\title{
Health-aware LPV-MPC based on System Reliability Assessment for Drinking Water Networks
}

\author{
Fatemeh Karimi Pour, Vicenç Puig and Gabriela Cembrano
}

\begin{abstract}
This paper proposes a health-aware model predictive control (MPC) for drinking water networks that includes an additional goal to extend the components and system reliability. The components and system reliability are incorporated in the MPC model as an extra parameter varying equation that considers the control action as a scheduling variable. The main goal of this work is to exhibit the advantage of taking into account system and component reliability, computed on-line by means of an LPV-MPC algorithm through an instance dedicated to DWNs. The proposed control approach allows the controller to accommodate to the parameter changes. By computing an estimation of the state variables during prediction, the MPC model can be modified considering the estimated state evolution at each time instant. Moreover, the solution of the optimization problem associate to the MPC problem is achieved by solving a series of Quadratic Programs (QP) at each sampling time. A small part of a real water network is used as a case study for illustrating the performance of the proposed approach.
\end{abstract}

\section{INTRODUCTION}

Drinking Water Networks (DWNs) are large-scale multisource/multi-node systems which must be reliable and flexible to deal with continuously varying conditions, as for example, unexpected changes in the demands or faults in some of the components [1]. DWN are multivariable dynamic constrained systems that are characterized by the interrelationship of several subsystems (actuators, tanks, intersection nodes, sources and consumer sectors). The application of control strategies by considering the system and components reliability becomes necessary to assure the quality of service. In order to increase the system reliability, anticipate the apparition of faults and reduce the operational costs, actuator health monitoring should be considered. Recently, system reliability has been taken into account in the system control process through a Prognosis and Health Management (PHM) framework. This is due to the fact that reliability is a standard procedure for estimating how long the system will perform its function accurately and can be used to predict future damages in the system given the state of its components [2]. According to the literature [3], [4], consumer demand satisfaction is the main objective of the operational control of a DWN. Additionally, its optimal management, that can be formulated as a multi-criteria problem, is a complicated challenge for water suppliers. To avoid these complications, Model Predictive Control (MPC) appears to be an efficient

This work has been partially funded by the Spanish State Research Agency (AEI) and the European Regional Development Fund (ERFD) through the projects DEOCS (ref. MINECO DPI2016-76493) and SCAV (ref. MINECO DPI2017-88403-R).This work has also been partially funded by AGAUR of Generalitat de Catalunya through the Advanced Control Systems (SAC) group grant (2017 SGR 482) ffkarimi, vpuig, cembrano\}eiri.upc.edu, method [5]. On the other hand, MPC has been recently proved as an adequate strategy for implementing healthaware control schemes because the MPC can predict the appropriate control actions to achieve optimal performance according to physical constraints and multi-objective cost functions. Pereira et al. in [6] proposed a MPC approach that distribute the loads among redundant actuators. Karimi Pour et al. [7] designed a health-aware MPC controller that includes a fatigue-based prognosis approach to minimize the damage of components.

The actuator reliability is usually modelled using exponential function of the control input [8]. On the other hand, the system reliability is determined from the combination of each actuator reliability taking into account the interconnection topology. Consequently, the system reliability has an exponential relation with the control input that induces a nonlinear behavior. Some studies on the DWN control are concentrated on the use of MPC to achieve an optimal management of the DWN system while maintaining the DWN reliability, e.g. by tuning the weights of the optimization problem according to a wear index [1] or by forcing constraints concerning actuator reliability [9]. One major drawback of the previous approaches to reliability-based MPC is that they consider the reliability at the actuator level but not at the system level taking into account the interconnection topology because of the non-linearity of the resulting constraints leading to the use of non-linear MPC. One way to deal with nonlinear MPC is to represent the process behavior by means of Linear Parameter Varying (LPV) models [10]. LPV models are a class of linear models whose state-space matrices depend on a set of time-varying parameters that can be measured/estimated on-line. The main advantage of LPV models is that the system nonlinearities are embedded in the varying parameters, which make the nonlinear system become a linear-like system with varying parameters [11].

This paper presents a health-aware LPV-MPC controller on the basis of PHM information provided by the on-line evaluation of the system reliability. The system reliability is integrated into the control algorithm using a LPV framework. The augmented model considering both the DWN and reliability models is represented as an LPV model. Thus, the control inputs are generated to fulfill the control objectives/constraints but at the same time to extend the reliability and lifespan of the system components. The first objective of this paper is to exhibit the advantage of taking into account system and component reliability, computed on-line in an LPV-MPC algorithm through an instance dedicated to DWNs. The second main contribution of this paper consists 
in designing an improved health-aware LPV-MPC strategy in order to formulate an optimization problem that exploits the functional dependency of scheduling variables and state vector to develop a prediction strategy with numerically attractive solution. This attractive solution is iteratively forced to an accurate solution, thereby avoiding the use of non-linear optimization.

The remainder of the paper is organized as follows. In Section II, the DWN control oriented modelling is introduced. The system reliability modeling is presented in Section III. The health-aware controller scheme based on an LPVMPC algorithm and the reliability model integration into the control algorithm are presented in Section IV. In Section V, results of applying the proposed control strategy to the DWN network as a case study are summarized. Finally, in Section VI, the conclusion of this work are drawn and some research lines for future work are proposed.

\section{MPC OF DRINKING WATER NETWORK}

\section{A. System description and modeling}

Several modeling methods dealing with DWNs have been proposed in the literature (see, e.g., [12]). In this paper, a control-oriented modeling approach that is based on a flow model is outlined following the principles introduced in [13]. Generally, a DWN is a network which comprises a set of pressurized pipes, water tanks at different elevations, a number of pumping stations and valves to manage water flows, pressure, and elevation to supply water to consumers. Consider a general DWN as represented by a directed graph $G(\nu, \varepsilon)$, where a set of elements, i.e., $n_{x}$ storage tanks, $n_{s}$ sources, $n_{d}$ sinks and $n_{q}$ intersection nodes are represented by $v \in \nu$ vertices, which are connected by $a \in \varepsilon$ links. Due to the network function, water is transported along the links by $n_{u}$ flow actuators (i.e., valves and pipes), passing through reservoirs or tanks, from specific origin locations to specific destination locations. The control-oriented model of DWN can be described by the following set of linear discrete-time difference-algebraic equations for all time instant $k \in \mathbb{Z}_{+}$:

$$
\begin{aligned}
x(k+1) & =A x(k)+B u(k)+B_{d} d_{m}(k), \\
0 & =E_{u} u(k)+E_{d} d_{m}(k),
\end{aligned}
$$

where the difference equations in (1a) describe the dynamics of the storage tanks, and the algebraic equations in (1b) consider the static relations in the network (i.e., mass balance at junction nodes). Furthermore, $x(k) \in \mathbb{R}^{n_{x}}$ is the volume of the storage tanks, $u(k) \in \mathbb{R}^{n_{u}}$ is the manipulated inputs (i.e. flows in pumps and valves) and $d_{m}(k) \in \mathbb{R}^{n_{m}}$ is the demanded flow considered as measured disturbances. $A, B, B_{d}, E_{u}, E_{d}$ and $C$ are time-invariant matrices of suitability dimensions dictated by the network topology.

\section{B. MPC formulation}

The aim of using MPC techniques for controlling water distribution networks is to compute, ahead of time, the input commands to achieve the optimal performance of the network according to a given set of control goals [13].
The control goal can formulated as the minimization of a convex multi-objective cost function which includes a term for minimizing the square difference between the predicted output and the set-point. The other objective of the controller for the DWN is to maintain the pumps and tanks under their bounds and extend the reliability of the system.

Considering the network mathematical model (1), the MPC controller design is based on minimizing the finite horizon cost

$$
J_{k}=\sum_{l=0}^{N_{p}}\left\|x(l+1 \mid k)-x_{r e f}(l+1)\right\|_{p, w_{1}}+\sum_{i=0}^{N_{p}-1}\|u(l \mid k)\|_{p, w_{2}},
$$

where $N_{p}$ is the prediction horizon. Furthermore, the subindex $p$ denotes the norm used (for this paper, the 2norm) and the weighting matrices $w_{1} \in \mathbb{R}^{n_{x} \times n_{x}}$ and $w_{2} \in$ $\mathbb{R}^{n_{u} \times n_{u}}$ are used to establish the priority of the different control objectives. Considering that the values of $x(0 \mid k)$ and $u(0 \mid k-1)$ are known at each time instant, the MPC optimization problem can be formulated as

$$
\min _{\mathbf{u}(k)} J_{k}(\mathbf{u}(k))
$$

subject to:

$$
\begin{aligned}
& x(l+1 \mid k)=A x(l \mid k)+B u(l \mid k)+B_{d} d_{m}(k), \\
& 0=E_{u} u(l \mid k)+E_{d} d_{m}(k), \\
& u(k), u_{k+1}, \ldots, u_{k+N_{p}-1}, \in \mathbb{U} \\
& x(k), u_{k+1}, \ldots, x_{k+N_{p}}, \in \mathbb{X} \\
& x(0 \mid k)=x(k),
\end{aligned}
$$

where $\mathbf{u}(k)=\left[u(k), u(k+1), \ldots, u\left(k+N_{p}-1\right)\right]^{T}$ is the decision sequence of controlled inputs.

\section{RELIABILITY ASSESSMENT}

\section{A. Failure rate and component reliability}

Definition 1: Reliability is the probability that components, units, types of equipment and systems will perform their predesignated function for a certain period of time under some operating conditions and specific environments [2].

Mathematically, reliability $R(t)$ is the probability that a system will be successful in the interval from time 0 to time $t$ :

$$
R(t)=P(T>t), \quad t \geq 0
$$

where $T$ is a nonnegative random variable which represents time-to-failure or failure time.

Definition 2: The unreliability of a component (or system) $R(t)$ is defined as the probability that the component or system experiences the first failure or has failed one or more times during the time interval 0 to time $t$.

Since the component is always in one of the two possible states (operational or failed), the following relation is satisfied

$$
F(t)+R(t)=1
$$

Several different functions have been used to characterize the reliability functions of time. Some of the more common 
reliability functions include the exponential, normal, lognormal and Weibull distributions [14]. In particular, engineering systems are organized to support varying amounts of loads characterized in terms of usage rate or occupied period. Several observational types of research have established that the function load strongly affects the component failure rate [15]. Hence, it is important to consider the load versus failure rate relationship when presenting system reliability evaluation. In the considered study, failure rates are obtained from actuators under different levels of load depending on the applied control input. One of the most used relations is based on assuming that actuator fault rates changes with the load through the following exponential law:

$$
\lambda_{j}=\lambda_{j}^{0} \exp \left(\beta_{j} u_{j}(k)\right), \quad j=1,2, \ldots, m
$$

where $\lambda_{j}^{0}$ represents the baseline failure rate (nominal failure rate) and $u_{j}(k)$ is the control action a time $k$ for the $j^{\text {th }}$ actuator. $\beta_{j}$ is a constant parameter that depends on the actuator characteristics.

In the useful period of life, the component can be characterized at a given time $t$ by a baseline reliability measure $R_{0}(t)$. In the following, $R_{0, j}(t)$ will be assigned to the reliability of the $j$-th actuator obtained under nominal operating conditions in the useful period of life such as:

$$
R_{0, j}(k)=\exp \left(-\lambda_{j}^{0} t\right), \quad j=1,2, \ldots, m
$$

Therefore, the component reliability of a system with the $j$-th component can be estimated by using the exponential function and depending on the baseline reliability level $R_{0, j}$ as

$$
R_{j}(k)=R_{0, j} \exp \left(-\int_{0}^{k} \lambda_{j}(s) d s\right), \quad j=1,2, \ldots, m
$$

where $\lambda_{j}(s)$ is the failure rate that is obtained from the $j$-th component under different levels of load.

\section{B. System reliability modeling}

For the analysis system reliability, it can be introduced a graphical network model in which it is possible to determine whether the system is working correctly by determining whether a successful path exists through the system between every source and demand. A path for the network is a set of components, such that if all the components in the set are successful, the system will be successful. A minimal path, $p_{s}$, is a set of components that make up a path, but the dismissal of any one component will generate the resulting set to not be a path. Therefore, the overall system reliability $R_{G}(k)$ is computed as

$$
R_{G}(k)=1-\prod_{j=1}^{s}\left(1-\prod_{i \in p_{s, j}} R_{j}(k)\right)
$$

where $j=1, \ldots, s$ is number of minimal paths. In order to integrate the reliability in the linear MPC model as an additional state variable, a transformation is required that allows to compute reliability in a linear-like form. The proposed transformation is based on using the logarithm (9). As stated in (5), the (9) can be rewritten as

$$
\log \left(Q_{G}(k)\right)=\log \left(\prod_{j=1}^{s}\left(1-\prod_{i \in p_{s, j}} R_{i}(k)\right)\right)
$$

by introducing a change of variable

$$
z_{j}(k)=1-\prod_{i \in p_{s, j}} R_{i}(k)
$$

equation (10) leads to

$$
\log \left(Q_{G}(k)\right)=\sum_{i \in p_{s, j}}^{s} \log \left(z_{j}(k)\right)
$$

According to (11), the $\log \left(z_{j}(k)\right)$ can be obtained as

$$
\log \left(z_{j}(k)\right)=\frac{\log \left(z_{j}(k)\right)}{\log \left(1-z_{j}(k)\right)} \sum_{i \in p_{s, j}} \log R_{i}(k)
$$

Then, by renaming $\beta_{j}(k)=\frac{\log \left(z_{j}(k)\right)}{\log \left(1-z_{j}(k)\right)}$ in (13), (10) can be expressed as

$$
\log \left(Q_{G}(k)\right)=\sum_{i \in p_{s, j}}^{s} \beta_{j}(k) \sum_{i \in p_{s, j}} \log R_{i}(k)
$$

Finally, the system unreliability of system can be estimated from the baseline of system unreliability as follows:

$$
\log \left(Q_{G}(k+1)\right)=\log \left(Q_{G}(k)\right)+\sum_{i \in p_{s, j}}^{s} \beta_{j}(k) \sum_{i \in p_{s, j}} \log R_{i}(k)
$$

\section{HEALTH-AWARE LPV-MPC}

This section addresses the inclusion of reliability information in the predictive control law as an additional state of the linear system. As described in Section III, the reliability of the DWN can be computed using the control input (pump commands) information. In order to include a new objective in the MPC that aims to increase the system reliability, the reliability model is approximated by means of a linear model (15). The new MPC model uses the following model

$$
x_{r}(k+1)=A_{r} x_{r}(k)+B_{r} u(k)+B_{r, d} d_{m}(k),
$$

where the state vector is defined as $x_{r}=$ $\left[x, \log \left(Q_{G}\right), \log \left(R_{1}\right), \ldots, \log \left(R_{i}\right)\right]^{T}$ and $y_{r}=\left[y, \log \left(Q_{G}\right)\right]^{T}$. 
The matrices of the augmented system are defined as

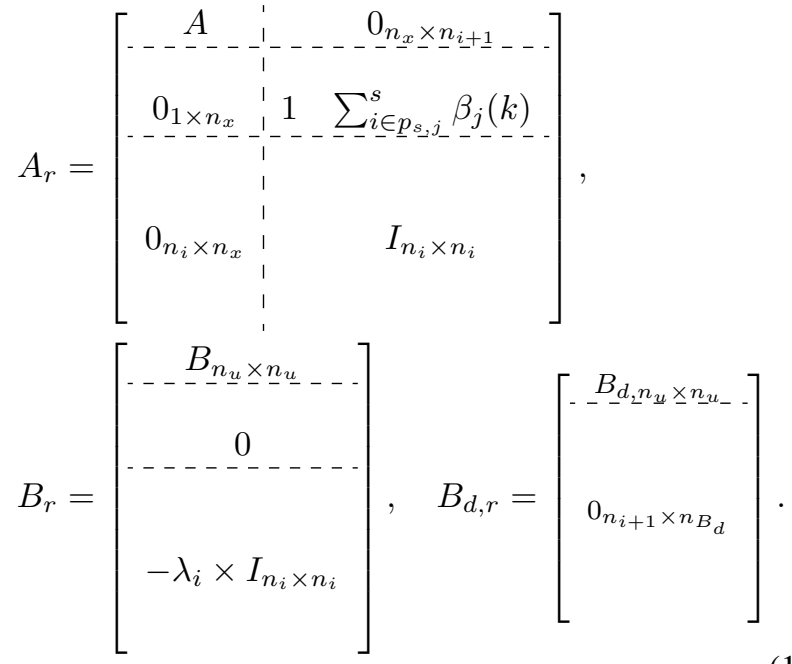

Therefore, the new MPC model (16) is a LPV model that has as scheduling variable the control action $u_{i}(k)$ associated to each actuator and state. The new MPC model (16) can not be evaluated before solving the optimization problem (3), because the future state sequence are not known. Indeed $x(l \mid k)$ depend not only on the future control inputs $u(k)$, but also on the future scheduling parameters, where for a general LPV model are not assumed to be known a priori but only to be measurable online at current time $k$. The idea is to find a solution to the problem (3) by solving an online optimization problem as a QP problem. In this paper, the solution for this problem is to transform the exact LPVMPC to an approximated form. This approximation is based on using an estimation of scheduling variables, $\hat{\theta}$ instead of using $\theta$. It means that the scheduling variables in the prediction horizon are estimated and used to update the matrices of the model used by the MPC controller. Thus, from the optimal control sequence $\mathbf{u}(\mathbf{k})$, it can be obtained the sequence of states and predicted parameters

$\tilde{\mathbf{x}}(k)=\left[\begin{array}{c}x(k+1) \\ x(k+2) \\ \vdots \\ x\left(k+N_{p}\right)\end{array}\right] \in \mathbb{R}^{N_{p, n_{x}}}, \quad \Theta=\left[\begin{array}{c}\hat{\theta}(k) \\ \hat{\theta}(k+1) \\ \vdots \\ \hat{\theta}\left(k+N_{p}-1\right)\end{array}\right] \in \mathbb{R}^{N_{p, n_{\theta}}}$

Therefore, with slight misuse of notation $f$ can be defined as: $\Theta(k)=f\left(\left[x^{T}(k) \quad \tilde{\mathbf{x}}^{T}(k)\right], \mathbf{u}(k)\right)$. The vector $\Theta(k)$ includes parameters from time $k$ to $k+N_{p}-1$ whilst the state prediction is accomplished for time $k+1$ to $k+N_{p}$.

Hence, by using the definitions (18), the predicted states can be simply formulated as follows

$$
\tilde{\mathbf{x}}(k)=\mathcal{A}(\Theta(k)) x(k)+\mathcal{B}(\Theta(k)) \mathbf{u}(k)+B_{r, d} d_{m}(k),
$$

where $\mathcal{A} \in \mathbb{R}^{n_{x} \times n_{x}}$ and $\mathcal{B} \in \mathbb{R}^{n_{x} \times n_{u}}$ are given by (20) and (21). By using (19) and augmented block diagonal weighting matrices $\tilde{w}_{1}=\operatorname{diag}_{N_{p}}\left(w_{1}\right)$ and $\tilde{w}_{2}=\operatorname{diag}_{N_{p}}\left(w_{2}\right)$, the cost function (2) with new additional objective that aims to maximize the system reliability can be rewritten in vector form as

$$
\begin{aligned}
& J(k)=\sum_{l=0}^{N_{p}-1}\left\|x(l+1 \mid k)-x_{r e f}(l+1)\right\|_{p, \tilde{w}_{1}}+ \\
& \|u(l+1 \mid k)\|_{p, \tilde{w}_{2}}-\left\|\log Q_{G}(l+1 \mid k)\right\|_{p, w_{3}},
\end{aligned}
$$

subject to:

$$
\begin{aligned}
& \tilde{\mathbf{x}}(k)=\mathcal{A}(\Theta(k)) x(k)+B_{r, d} d_{m}(k), \mathcal{B}(\Theta(k)) \mathbf{u}(k) \\
& 0=E_{u} u(l \mid k)+E_{d} d_{m}(k), \\
& \log Q_{G}(l+1 \mid k)=\tilde{\mathbf{x}}_{4}(k) \\
& u(k), u_{k+1}, \ldots, u_{k+N_{p}-1} \in \mathbb{U} \\
& x(k), u_{k+1}, \ldots, x_{k+N_{p}-1} \in \mathbb{X} \\
& x(0 \mid k)=x(k),
\end{aligned}
$$

while the reliability-aware objective with the corresponding weight $w_{3}$ is appended in the LPV-MPC cost function to maximize the system reliability. Since the predicted states $\Theta(k)$ in (19) are linear in control inputs $\mathbf{u}(k)$, the optimization problem can be solved as a QP problem, that is significantly easier than solving a nonlinear optimization problem.

This idea leads to the following iterative approach at each discrete time instant $k$ :

- In the first iteration, the problem (3) is solved as a linear problem due to the quasi-LPV model (16) is replaced by the LTI model that is obtained considering $\theta(0 \mid l) \simeq$ $\theta(1 \mid l) \simeq \theta(2 \mid l) \simeq \ldots \simeq \theta\left(N_{p}-1 \mid l\right)$ along the prediction horizon $N_{p}$.

- The sequence of the scheduling variables $\Theta(k)$ is repetitively steered to its optimal amount $\Theta^{*}(k)=$ $f\left(\tilde{\mathbf{x}}^{*}(k), \mathbf{u}^{*}(k)\right)$, when $\tilde{\mathbf{x}}^{*}(k)$ and $\mathbf{u}^{*}(k)$ refer the input and state sequences related to the optimal solution.

- The optimal amount $\Theta^{*}(k)$ obtained by solving the optimization problem in iteration step $l$ when $\Theta(k)$ replaced by $\Theta_{i}(k)$, and by creating a new premise sequence from the result of the optimal state sequence $\tilde{\mathbf{x}}_{l}(k)$ as $\Theta_{l+1}(k)=f\left(\tilde{\mathbf{x}}_{l}(k), \mathbf{u}_{l}(k)\right)$.

- The premise variable for the next iteration $\Theta_{0}(k+1)$ is determined when using $\tilde{\mathbf{x}}_{l}(k)$ and $\mathbf{u}_{l}(k)$, i.e., $\Theta_{0}(k+$ $1)=f\left(\tilde{\mathbf{x}}_{l}(k), \mathbf{u}_{l}(k)\right)$.

\section{Application ExAmple}

\section{A. Case study}

For the sake of illustration, a portion of the Barcelona DWN reported [16] is proposed. Regarding the DWN reliability study, sinks, sources, pipelines and tanks are considered perfectly reliable whereas this is not the case of active elements [3]. The case study includes two sources of water and four demand sector (see Fig. 1). It is expected that the demand forecast $\left(d_{m}\right)$ at each sink is known and that every single source can provide this required water demand Fig. 2. Moreover, it is assumed that the volume of the tanks should track a specified set-point. 


$$
\mathcal{A}(\Theta(k))=\left[\begin{array}{c}
I \\
A(\hat{\theta}(k)) \\
A(\hat{\theta}(k+1)) A(\hat{\theta}(k)) \\
\vdots \\
A\left(\hat{\theta}\left(k+N_{p}-1\right)\right) A\left(\hat{\theta}\left(k+N_{p}-2\right)\right) \ldots A(\hat{\theta}(k))
\end{array}\right]
$$

and

$$
\mathcal{B}(\Theta(k))=\left[\begin{array}{ccccc}
0 & 0 & 0 & \ldots & 0 \\
B(\hat{\theta}(k)) & 0 & 0 & \ldots & 0 \\
A(\hat{\theta}(k+1)) B(\hat{\theta}(k)) & B(\hat{\theta}(k+1)) & 0 & \ldots & 0 \\
\vdots & \vdots & \ddots & \ddots & \vdots \\
A\left(\hat{\theta}_{k+N_{p}-1}\right) \ldots A(\hat{\theta}(k+1)) B(\hat{\theta}(k)) & A\left(\hat{\theta}_{k+N_{p}-1}\right) \ldots A(\hat{\theta}(k+2)) B(\hat{\theta}(k+1)) & \ldots & \left.B\left(\hat{\theta}_{k+N_{p}-1}\right)\right) & 0
\end{array}\right] .
$$

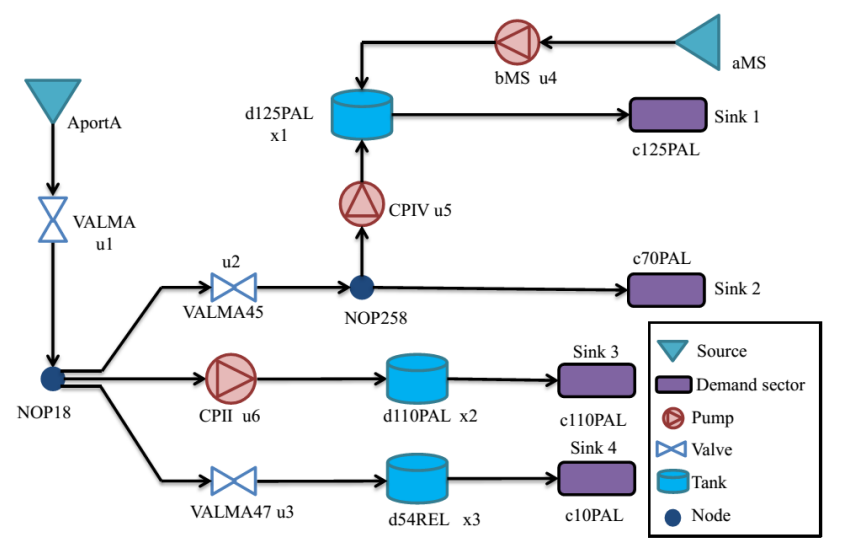

Fig. 1. Drinking water network diagram (Three-tanks).

\section{B. Results and analysis}

The reliability-aware LPV-MPC formulation proposed in previous section has been applied to a simulation model of DWN in Fig. 1. Firstly, system components must be identified. Then, the minimal path sets should be determined. By considering all the paths from all the sources to the sinks, the combination of all flow paths should follow the functional requirements necessary to satisfy the consumer demands. A
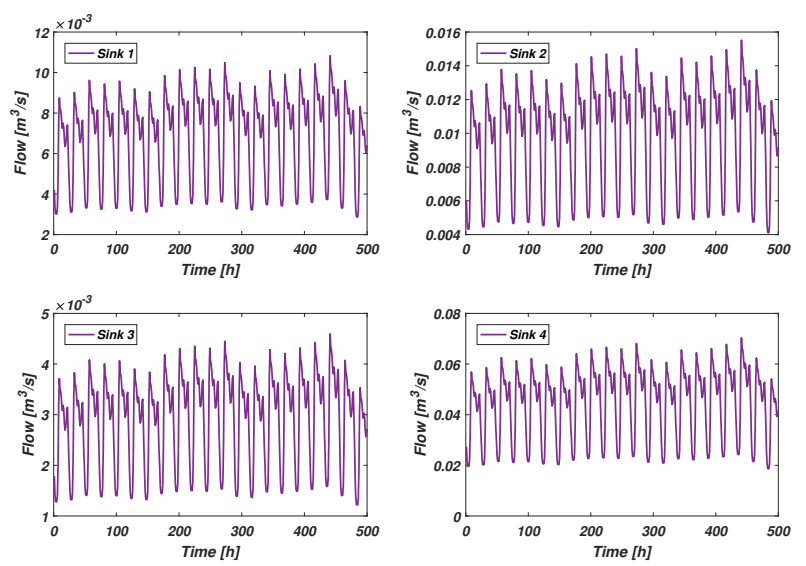

Fig. 2. Drinking water demand for four sink. minimal path set is composed by those components which allow a flow path between sources and sinks, such as pipes, tanks and pumps. Based on this analysis, the following list of each minimal paths is presented in Table I. There are five minimal path sets in the system of Fig. 2. The reliability of each minimal path set depends on the reliability of its components. Tanks and pipes are supposed perfectly reliable. Table II provides the simulation parameters used. Figure 3 shows the evaluation of the valves and pumps commands results that obtained using the health-aware LPV-MPC in simulation considering the reliability-aware objective. Figure 4 presents the tracking response of the control algorithm. The system reliability prediction of the DWN obtained from the proposed controller with and without the reliabilityaware objective are presented in Fig. 5. According to these results, it can be observed that with the use of reliabilityaware objective in the MPC, the reliability of the network is better preserved compared to the case that the reliability is not considered in the MPC design. Figure 5 show that the system reliability is improved about $9.06 \%$ in the LPVMPC controller with the reliability objective while keeping the set-point tracking performance.
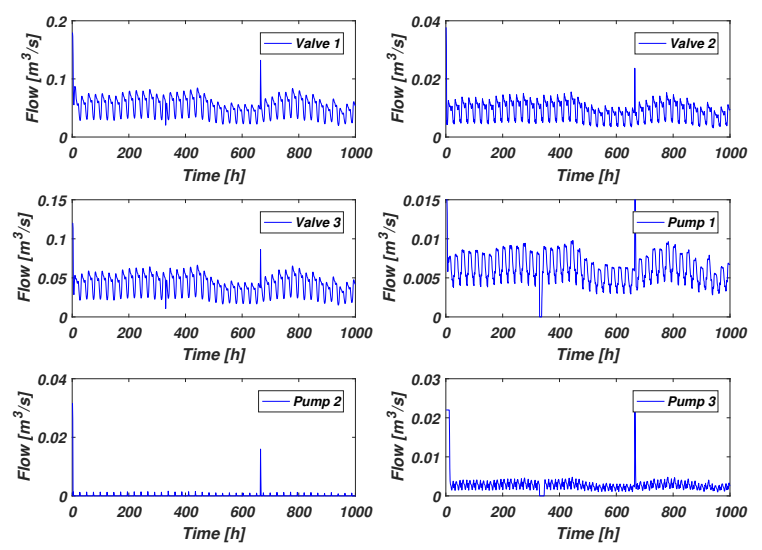

Fig. 3. Evaluation of the valves and pumps results.

\section{CONCLUSIONS}

This paper has proposed a health-aware LPV-MPC strategy based on the maximization the whole reliability of 
TABLE I

SUCCESS MINIMAL PATHS OF THE BARCELONA DWN

\begin{tabular}{l||l} 
Path & Component Set \\
\hline \hline & \\
$P_{1}$ & $\{$ Aport $A, V A L M A, V A L M A 47, c 10 C O R\}$ \\
$P_{2}$ & $\{$ Aport $A, V A L M A, C P I I, c 110 P A P\}$ \\
$P_{3}$ & $\{$ Aport $A, V A L M A, V A L M A 45, c 70 P A L\}$ \\
$P_{4}$ & $\{$ Aport $A, V A L M A, V A L M A 47, C P I V, c 125 P A L\}$ \\
$P_{5}$ & $\{a M S, b M S, c 125 P A L\}$
\end{tabular}

TABLE II

SIMULATION PARAMETERS.

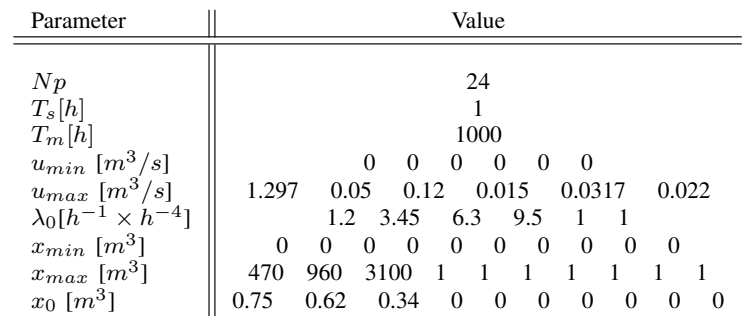

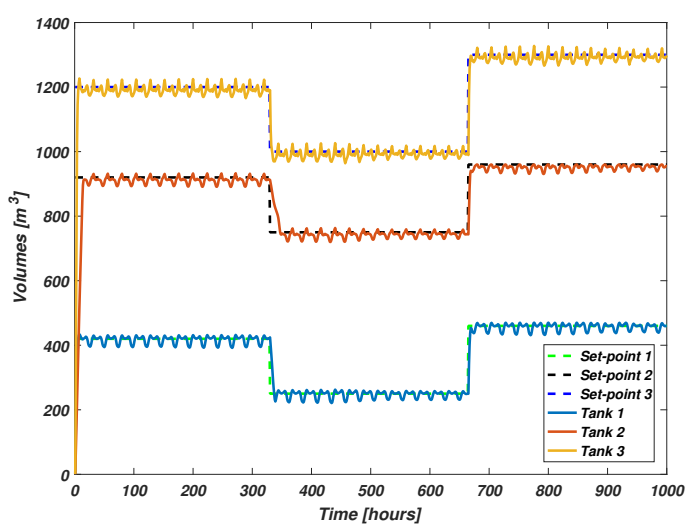

Fig. 4. Evaluation of tracking references of tanks.

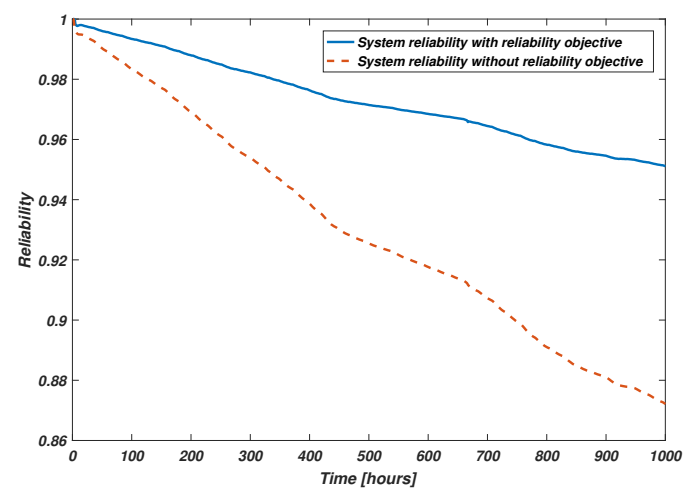

Fig. 5. Evaluation of system reliability.
DWN network. The system reliability is obtained as a function of control action via the reliability assessment of each component. The model of the system reliability is obtained as a function of control action with a nonlinear term that is transformed in a linear-like form via the LPV framework. Then, the maximization of the system reliability has achieved by including it in the objective function and as an additional state in the MPC model. The new healthaware LPV-MPC approach is efficiently solved iteratively by a series of QP problems that uses an update MPC model up dated via the scheduling parameters calculated at each time instant. The model prediction in the MPC horizon is obtained using the previous sequence of scheduling variables. The results obtained show that the system reliability of the DWN network is maximized with the proposed controller.

\section{REFERENCES}

[1] J. M. Grosso, C. Ocampo-Martínez, V. Puig, A service reliability model predictive control with dynamic safety stocks and actuators health monitoring for drinking water networks, in: Decision and Control (CDC), 2012 IEEE 51st Annual Conference on, IEEE, 2012, pp. $4568-4573$.

[2] I. Gertsbakh, Theory of reliability with applications to preventive maintenance (2000).

[3] P. Weber, C. Simon, D. Theilliol, V. Puig, Fault-tolerant control design for over-actuated system conditioned by reliability: a drinking water network application., IFAC Proceedings Volumes 45 (20) (2012) 558563.

[4] D. Robles, V. Puig, C. Ocampo-Martinez, L. E. Garza-Castañón, Reliable fault-tolerant model predictive control of drinking water transport networks, Control Engineering Practice 55 (2016) 197-211.

[5] J. M. Maciejowski, Predictive control: with constraints, Pearson education, 2002.

[6] E. B. Pereira, R. K. H. Galvão, T. Yoneyama, Model predictive control using prognosis and health monitoring of actuators, in: Industrial Electronics (ISIE), 2010 IEEE International Symposium on, IEEE, 2010, pp. 237-243.

[7] F. Karimi Pour, V. Puig Cayuela, C. Ocampo-Martínez, Healthaware model predictive control of pasteurization plant, in: ACD'1613th European Workshop on Advanced Control and Diagnosis 17-18 November 2016, Lille, France, 2016.

[8] A. Chamseddine, D. Theilliol, I. Sadeghzadeh, Y. Zhang, P. Weber, Optimal reliability design for over-actuated systems based on the mit rule: Application to an octocopter helicopter testbed, Reliability Engineering \& System Safety 132 (2014) 196-206.

[9] J. C. Salazar, P. Weber, R. Sarrate, D. Theilliol, F. Nejjari, Mpc design based on a dbn reliability model: Application to drinking water networks, IFAC-PapersOnLine 48 (21) (2015) 688-693.

[10] P. Bumroongsri, S. Kheawhom, Mpc for lpv systems based on parameter-dependent lyapunov function with perturbation on control input strategy, Engineering Journal 16 (2) (2012) 61.

[11] F. Karimi Pour, V. Puig Cayuela, C. Ocampo-Martínez, Comparative assessment of lpv-based predictive control strategies for a pasteurization plant, in: 4th-2017 International Conference on Control, Decision and Information Technologies, 2017, pp. 1-6.

[12] L. Mays, Urban stormwater management tools, McGraw Hill Professional, 2004.

[13] C. Ocampo-Martinez, V. Puig, G. Cembrano, J. Quevedo, Application of predictive control strategies to the management of complex networks in the urban water cycle [applications of control], IEEE Control Systems 33 (1) (2013) 15-41.

[14] R. Jiang, A. K. Jardine, Health state evaluation of an item: A general framework and graphical representation, Reliability Engineering \& System Safety 93 (1) (2008) 89-99.

[15] J. C. Salazar, P. Weber, F. Nejjari, R. Sarrate, D. Theilliol, System reliability aware model predictive control framework, Reliability Engineering \& System Safety 167 (2017) 663-672.

[16] G. Cembrano, J. Quevedo, M. Salamero, V. Puig, J. Figueras, J. Mart1, Optimal control of urban drainage systems. a case study, Control engineering practice 12 (1) (2004) 1-9. 